\title{
Bilateral Primary T cell Intraocular Lymphoma: Diagnostic Utility of Autofluorescence and Electroretinography
}

\author{
Chancellor JR ${ }^{1}$, Jones $\mathrm{MH}^{1}$, Siddiqui $\mathrm{MZ}^{3}$, Uwaydat SH${ }^{1}$, Lobo RH${ }^{2}$, \\ Brown $\mathrm{H}^{1,2}$ and Sallam $\mathrm{AB}^{1 *}$ \\ ${ }^{1}$ Jones Eye Institute, University of Arkansas for Medical Sciences, US \\ 2Department of Pathology and Laboratory Services, University of Arkansas for \\ Medical Sciences, US \\ ${ }^{3}$ College of Medicine, University of Arkansas for Medical Sciences, US
}

*Corresponding author: Ahmed B Sallam, Jones Eye Institute, University of Arkansas for Medical Sciences, Arkansas, US, E-mail: asallam@uams.edu

\section{Abstract}

Background: Primary intraocular lymphoma is a rare malignancy. Within the subtypes of intraocular lymphoma, $\mathrm{T}$ cell intraocular lymphoma is seen much less often than B cell intraocular lymphoma, and when it occurs it is often a component of a primary systemic lymphoma. Intraocular lymphoma is typically diagnosed with clinical findings and biopsy. Here we demonstrate the diagnosis of $\mathrm{T}$ cell primary intraocular lymphoma in a fellow, asymptomatic eye of a patient with auto fluorescence and electroretinography who had primary intraocular lymphoma.

Case Presentation: A 73-year-old female presented to the Jones Eye Institute due to blurred vision of the right eye. Fundus examination revealed creamy yellow sub retinal lesions as well as vitritis. After an extensive work up and multiple vitreous biopsies, she was diagnosed with $\mathrm{T}$ cell intraocular lymphoma. Auto fluorescence imaging showed signs of possible involvement of the fellow eye that were not visible clinically, which was later confirmed with electro diagnostic retinal testing. No evidence of systemic lymphoma was initially demonstrated on brain imaging, systemic imaging, or lumbar puncture.

Conclusion: Intraocular lymphoma poses a diagnostic challenge to the treating physician, particularly if not associated with systemic disease. Definitive diagnosis depends on demonstration of malignant lymphoma cells in ocular specimens. Auto fluorescence and electro diagnostic retinal testing can assist in the diagnosis of disease in fellow eyes of unilaterally presenting cases.

Keywords: Chorioretinitis; Primary Intraocular Lymphoma; T Cell Intraocular Lymphoma; Cytopathology; Vitrectomy 


\section{Open Access Journal of Ophthalmology}

Abbreviations: IOL: Intraocular Lymphoma; PET: Positron Emission Tomography; ERG: Electroretinogram.

\section{Background}

T cell intraocular lymphoma (IOL) is a rare subtype of primary intraocular lymphoma. The most common type in this group of malignancies is high grade B cell intraocular lymphoma [1]. Other rare types include natural killer $\mathrm{T}$ cell lymphomas [2]. Due to the rarity of this disease, only case reports and small series of T cell IOL have been reported. There have been reported cases of primary $\mathrm{T}$ cell intraocular lymphoma, ocular-CNS T cell lymphoma, as well as ocular-systemic T cell lymphoma, with mycosis fungoides subtype being the most common systemic subtype [1]. Diagnosis is typically made with a combination of clinical findings and vitreous sampling with cytopathology. Here we present a case of primary $\mathrm{T}$ cell intraocular lymphoma diagnosed with clinical findings and cytopathologic diagnosis in one eye. Subclinical $\mathrm{T}$ cell lymphoma was diagnosed with auto fluorescence and electroretinogram in the fellow eye.

\section{Case Presentation}

A 73-year-old female was referred to our institution due to a three-month history of progressively blurred vision of the right eye. She had been seen by an outside retina specialist and was found to have creamy yellow sub retinal lesions. Her workup at the outside facility included aspiration of aqueous fluid, which tested negative for toxoplasmosis, HSV, and VZV. She also had a negative FTA-ABS test for syphilis, a negative Quantiferon gold test for tuberculosis, and negative serum toxoplasmosis IgM and IgG. A chest X-ray showed no abnormalities. She had been treated with oral valacyclovir for presumed viral retinitis for two weeks with no improvement in symptoms and extension of the retinitis was noted clinically. It was at this time she was referred to our institution.

On initial examination at our facility, it was found that the patient's Snellen visual acuity was 20/50 OD and $20 / 25$ OS. Her slit lamp exam was significant for trace anterior chamber cell in the right eye. Fundus examination showed vitreous cells with $2+$ vitreous haze and 2 yellow subretinal lesionsin the right eye (Figure 1A). The left eye clinical examination and auto fluorescence were unremarkable (Figure 1B, 1D).
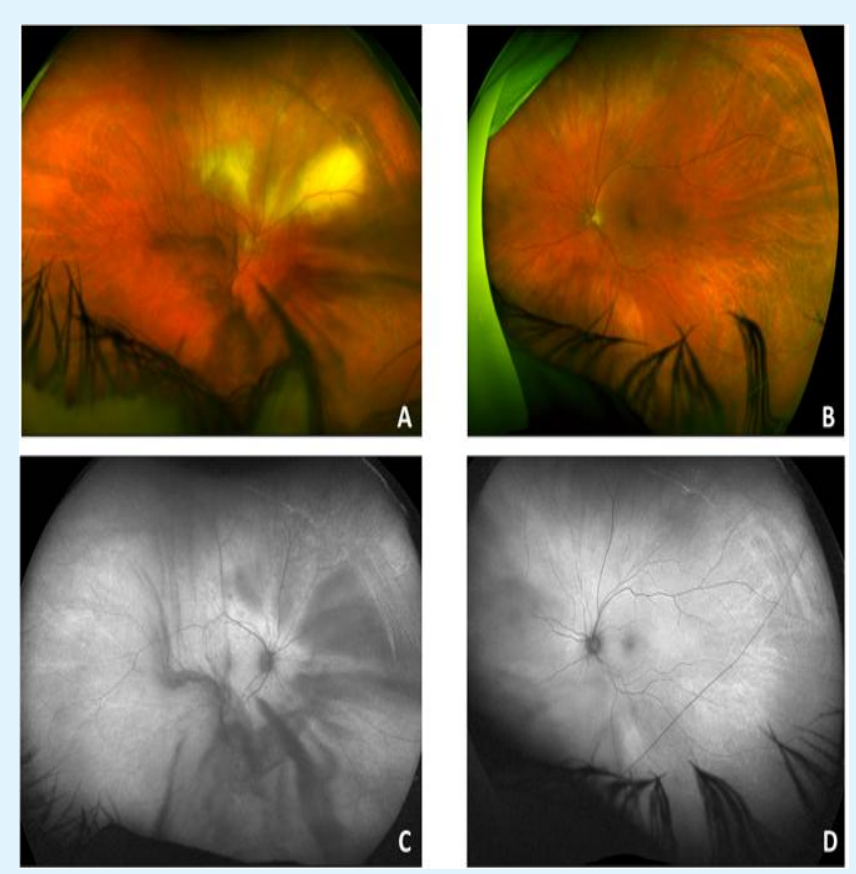

Figure 1: Retinal imaging at initial presentation.

Figure 1A: Color fundus photograph of the right eye showing areas of yellow sub retinal infiltrates above the optic disc and superior arcade with small areas of intra retinal hemorrhages and vitreous opacities.

Figure 1C: Auto fluorescence image of the right eye showing mixed hyper- and hypo-auto fluorescence superiorly and temporally corresponding with the infiltrates.

Figure 1B + 1D: Color fundus photograph and auto fluorescence image of the left eye appears unremarkable.

At this time, the patient underwent aspiration of vitreous fluid as well as an intravitreal injection of clindamycin, due to concern for toxoplasmosis. She was started on oral treatment for toxoplasmosis with oral trimethoprim/sulfamethoxazole and clindamycin. The vitreous fluid was sent for HSV, VZV, and toxoplasmosis testing, which were negative. A repeat aspiration of vitreous fluid was sent for cytopathologicalexamination and was found to be suspicious for lymphoma. At this time, a diagnostic pars plana vitrectomy was scheduled. Microscopic analysis of the vitrectomy sample showed large, highly atypical lymphoid cells displaying irregular nuclear membrane contours and multiple prominent nucleoli (Figure 2). 


\section{Open Access Journal of Ophthalmology}

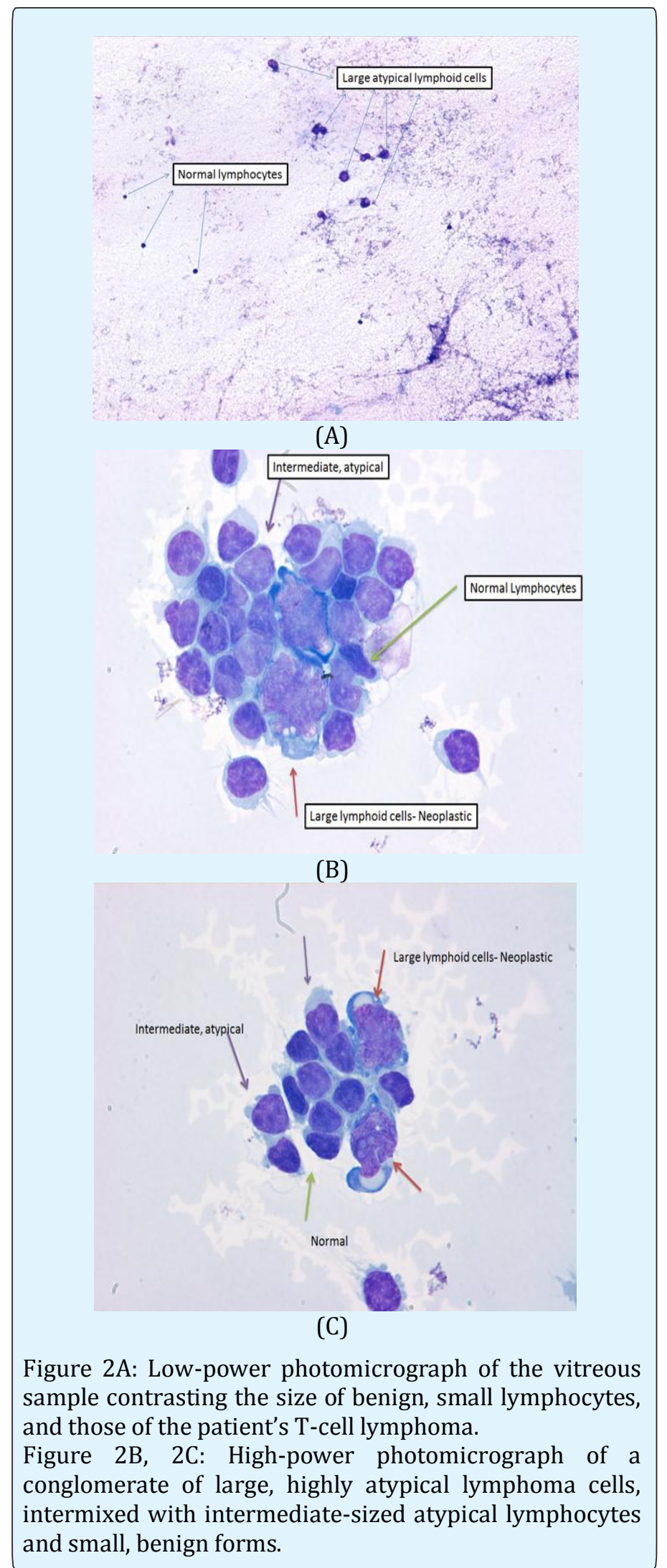

Flow cytometric analysis revealed a large population of lymphoid cells (97\%) comprised of CD3-positive T cells of the T-helper subtype (CD4-positive) with appropriate coexpression of CD2 and CD5, but with significant aberrant loss of CD7; thus the morphologic and immunophenotypic findings of the vitreous fluid examination were consistent with T cell lymphoma.

Repeat imaging was performed after vitrectomy. Although symptoms were only present in the right eye, auto fluorescence imaging showed the presence of new hyper-auto fluorescent spots in the left eye, concerning for the development of bilateral disease (Figure 3).

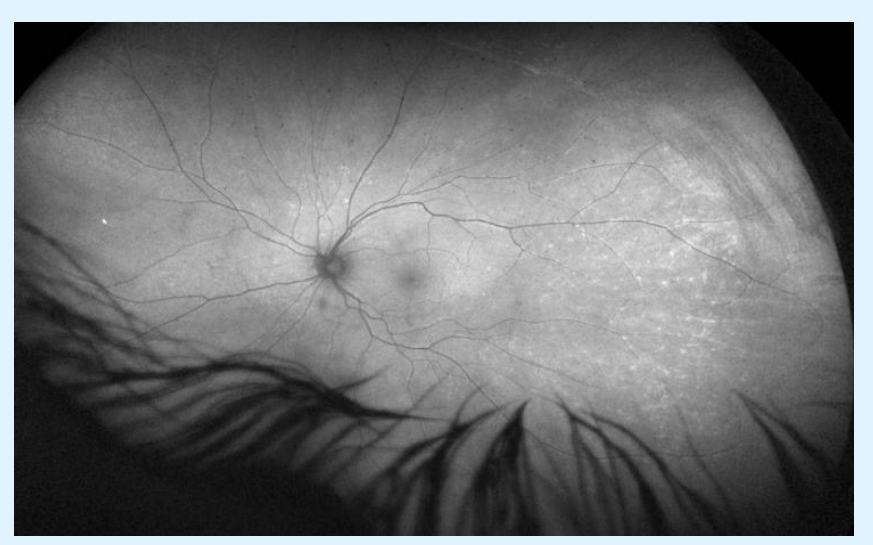

Figure 3: Autofluorescenceimage of left eye showing multiple hyper-autofluorescent punctate lesions noted temporally.

The patient was referred to an oncologist and underwent a systemic workup. Her brain MRI showed no evidence of CNS disease. She underwent a lumbar puncture which showed no signs of malignant cells. A full body positron emission tomography (PET) scan showed no systemic involvement.

The course of treatment recommended by oncology depended on demonstration of bilateral versus unilateral disease. If the disease is definitively in both eyes, systemic methotrexate treatment is recommended, following the CNS lymphoma protocol. If the disease is only unilateral, local treatment with intravitreal methotrexate is recommended. To evaluate for evidence of bilateral disease, a small sample of vitreous was aspirated from the left eye. Flow cytometry on this specimen revealed no abnormal $\mathrm{T}$ cell populations. This was not unexpected given the small sample size of vitreous aspirate compared to vitrectomy with biopsy. A study showed that only $7.4 \%$ of patients were diagnosed with vitreous tap, with $63 \%$ of 


\section{Open Access Journal of Ophthalmology}

patients demonstrating at least one false negative and most patients requiring 2 procedures for diagnosis [3]. The patient declined vitrectomy as she was asymptomatic in that eye.

Full field electroretinogram (ERG) revealed that the rod and cones had normal amplitudes but the implicit times were delayed in both eyes for both rods and cones. The rod implicit time in the right eye was $57.5 \mathrm{~ms}$ and $58 \mathrm{~ms}$ in the left eye, with the normal range being $43-52 \mathrm{~ms}$. The cone implicit time in the right eye was $37.5 \mathrm{~ms}$ and $36.5 \mathrm{~ms}$ in the left eye, with the normal range being $25-31 \mathrm{~ms}$. Multifocal ERG showed that the amplitudes of the perifoveal wavelets were reduced in both eyes, with the right eye being more significantly affected than the left.

Due to the above findings and the overall clinical picture, it was decided that the patient likely had bilateral disease. Treatment options were discussed with the patient, and she declined systemic treatment, instead opting for a course of bilateral methotrexate intravitreal injections. Improvement of the lesions was observed clinically and on auto fluorescence in both eyes after several months (Figure 4). She was given 25 injections OD and 13 injections OS (withheld due to macular cysts and MTX toxicity) over the course of one year. Two months after her final injection, she presented to the hospital with weakness and lethargy. MRI detected new basal ganglia mass and midline shift. She was intubated and decision was made for comfort care measures.
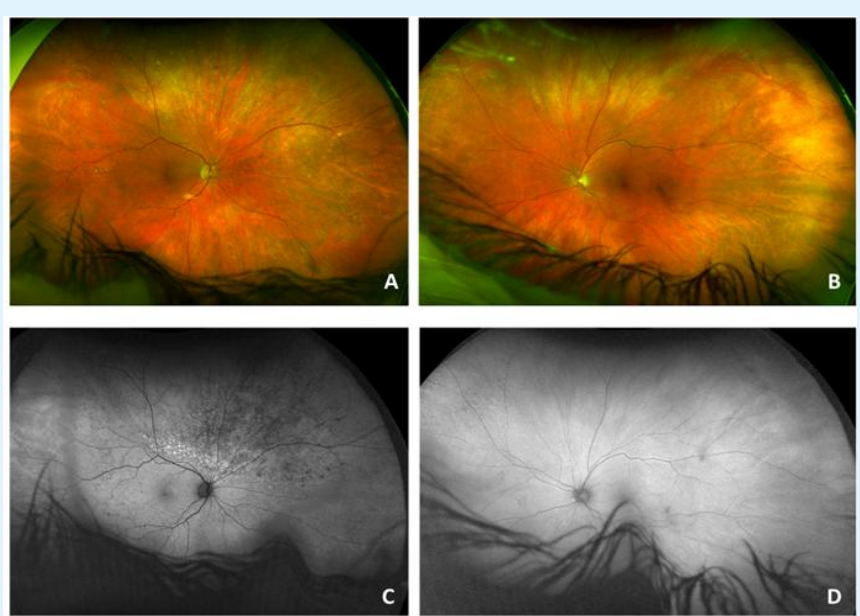

Figure 4: Repeat retinal imaging after five months of treatment in the right eye and two months of treatment in the left eye. Improvement observed on color and auto fluorescence $(A, C)$ in the right eye. No change in color fundus photograph in the left eye (B), but there was improvement observed on the auto fluorescence image of the left eye (D).

\section{Conclusion}

$\mathrm{T}$ cell intraocular lymphoma is a rare subtype of primary intraocular lymphoma. Approximately $80 \%$ of all published cases of $\mathrm{T}$ cell intraocular lymphoma had extraocular systemic lymphoma, mycosis fungoides, or primary CNS lymphoma with intraocular involvement [4].

The differential diagnosis in this patient at presentation included mainly CMV retinitis or herpetic acute retinal necrosis, toxoplasma chorioretinitis, tuberculosis or syphilis chorioretinitis, primary systemic inflammatory disorders (particularly Behcet's disease) and intraocular lymphoma.

Our patient developed a progressive necrotizing chorioretinitis with significant vitritis. The initial unilateral presentation and the absence of associated systemic features as skin and mucous membranes ulcers made the diagnosis of Behcet's disease unlikely. Chronicity, lack of response to anti-viral treatment and non-revealing aqueous PCR analysis for herpes viruses, interferon gamma release assay for TB and syphilis serology made the diagnosis of viral, TB or syphilitic chorioretinitis unlikely. Despite a negative toxoplasma PCR from aqueous, the facts that the sensitivity of vitreous PCR for toxoplasmosis is only approximately $60 \%$ and that toxoplasma retinitis could be multifocal in the elderly led to consideration and treatment of toxoplasma retinitis [5]. However, lack of a positive toxoplasma PCR on repeat testing increased the suspicion of lymphoma and prompted diagnostic vitrectomy for repeat sampling of vitreous fluid.

There are 2 main points in this case that deserve attention. First establishing a definitive diagnosis of intraocular lymphoma can be challenging; in many cases intraocular lymphoma mimics viral or multifocal toxoplasma retinitis [6]. Diagnosis requires high index of suspicion and usually involves repeat vitreous sampling or chorioretinal biopsy and the use of additional diagnostic techniques such as immunohistochemistry and flow cytometric analysis to characterize and quantitate the proportion of lymphocytesexpressing or lacking expression of specific markers gene rearrangement studies with polymerase chain reaction, and more recently demonstration of elevated vitreous interleukin 10/ interleukin 6 ratio [7-9].

Second, primary intraocular lymphoma typically presents bilaterally $(71 \%)$ compared to unilaterally (29\%) [1]. Therefore, in a patient presenting with unilateral symptoms, clinicians should have a high index 


\section{Open Access Journal of Ophthalmology}

of suspicion for subclinical bilateral disease. In our patient, the presence of hyper-autofluorescent fundal lesions in the asymptomatic fellow eye prompted ERG testing and the diagnosis of bilateral disease was made. Given the low yield of vitreous tap and risks of vitrectomy in any asymptomatic eye, these methods may offer useful, non-invasive adjuncts for diagnosis of bilateral disease. The diagnosis of unilateral versus bilateral disease is clinically relevant for determining local versus systemic therapy.

In summary, as demonstrated in our case, intraocular lymphoma may be clinically indistinguishable at presentation from viral or multifocal retinitis. Auto fluorescence and electro diagnostic studies may diagnose subtle involvement of the fellow eye in intraocular lymphoma initially presenting as unilateral disease.

\section{References}

1. Chaput F, Amer R, Baglivo E, Touitou V, Kozyreff A, et al. (2017) Intraocular T-cell Lymphoma: Clinical Presentation, Diagnosis, Treatment, and Outcome. Ocul Immunol Inflamm 25(5): 639-648.

2. Maruyama K, Kunikata H, Sugita S, Mochizuki M, Ichinohasama R, et al. (2015) First case of primary intraocular natural killer t-cell lymphoma. BMC Ophthalmology 15: 169.

3. Dalal M, Casady M, Moriarty E, Faia L, Nussenblatt R, et al. (2014) Diagnostic procedures in vitreoretinal lymphoma. Ocul Immunol Inflamm 22(4): 270-276.
4. Coupland S, Anastassiou G, Bornfeld N, Hummel M, Stein H (2005) Primary Intraocular lymphoma of Tcell Type: report of a case and review of the literature. Graefe's Arch Clin Exp Ophthalmol 243(3): 189-197.

5. de Boer JH, Verhagen C, Bruinenberg M, Rothova A, de Jong PT, et al. (1996) Serologic and polymerase chain reaction analysis of intraocular fluids in the diagnosis of infectious uveitis. Am J Ophthalmol 121(6): 650658.

6. Sagoo M, Mehta $\mathrm{H}$, Cohen $\mathrm{V}$, Amin S, Plowman $\mathrm{P}$ (2014) Primary intraocular lymphoma. SurvOphthalmol 59(5): 503-516.

7. Raparia K, Chang CC, Chevez-Barrios P (2009) Intraocular lymphoma: diagnostic approach and immunophenotypic findings in vitrectomy specimens. Arch Pathol Lab Med 133(8): 1233-1237.

8. Katai N, Kuroiwa S, Fujimori K, Yoshimura N (1997) Diagnosis of intraocular lymphoma by polymerase chain reaction. Graefe's Arch Clin Exp Ophthalmol 235(7): 431-436.

9. Wang Y, Shen D, Wang VM, Sen HN, Chan C (2011) Molecular biomarkers for the diagnosis of primary vitreoretinal lymphoma. Int J Mol Sci 12(9): 56845697.

10. Goldey SH, Stern GA, Oblon DJ, Mendenhall NP, Smith LJ, et al. (1989) Immunophenotypic characterization of an unusual T-cell lymphoma presenting as anterior uveitis. A clinicopathologic case report. Arch Ophthalmol 107(9): 1349-1353. 